\title{
PERFIL DOS PROFISSIONAIS DA ATENÇÃO PRIMÁRA Á SAÚde, vINCUlados aO CURSO DE ESPECIALIZAÇÃO EM SAÚDE DA FAMILLA UNA-SUS NO RIO GRANDE DO SUL
}

\author{
PROFILE OF PRIMARY HEALTH CARE PROFESSIONALS \\ LINKED TO UNA-SUS SPECIALIZATION PROGRAM IN \\ FAMILY HEALTH IN RIO GRANDE DO SUL
}

Recebido em: 13 de setembro de 2019

Aprovado em: 02 de dezembro de 2019

Sistema de Avaliação: Double Blind Review

RCO |a. 12 |v. 1 |p. 04-26|jan./abr. 2020

DOI: https://doi.org/10.25112/rco.v1i0.1639

\section{Giovani Sturmer gstr@outlook.com}

Doutor em Ciências da Saúde pela Fundação Universidade Federal de Ciências da Saúde de Porto Alegre (Porto Alegre/Brasil). Professor do Curso de Fisioterapia da Universidade de Cruz Alta (Cruz Alta/Brasil). Currículo Lattes: http://lattes.cnpq.br/6665244655294794

Maria Eugênia Bresolin Pinto meugeniap2@gmail.com

Doutora em Epidemiologia pela Universidade Federal do Rio Grande do Sul (Porto Alegre/Brasil). Professora do Departamento de Saúde Coletiva da Fundação Universidade Federal de Ciências da Saúde de Porto Alegre (Porto Alegre/Brasil). Currículo Lattes: http://lattes.cnpq. br/9611281263934506

\section{Monica Maria Celestina de Oliveira monica@ufcspa.edu.br}

Doutora em Epidemiologia pela Universidade Federal do Rio Grande do Sul (Porto Alegre/Brasil). Professora do Departamento de Saúde Coletiva da Fundação Universidade Federal de Ciências da Saúde de Porto Alegre (Porto Alegre/Brasil). Currículo Lattes: http://lattes.cnpq. br/0054153725587996

\section{Alessandra Dahmer adahmer@gmail.com}

Doutora em Ciência da Computação pela Universidade Federal do Rio Grande do Sul (Porto Alegre/Brasil). Professora do Departamento de Ciências Exatas e Sociais Aplicadas da Fundação Universidade Federal de Ciências da Saúde de Porto Alegre (Porto Alegre/Brasil).

Currículo Lattes: http://lattes.cnpq.br/9092647951099642

\section{Airton Tetelbom Stein astein@ufcspa.edu.br}

Doutor em Medicina pela Universidade Federal do Rio Grande do Sul (Porto Alegre/Brasil). Professor Titular do Departamento de Saúde Coletiva da Fundação Universidade Federal de Ciências da Saúde de Porto Alegre (Porto Alegre/Brasil), Médico de Família e Comunidade. Currículo Lattes: http://lattes.cnpq.br/2762761928704612

\section{Rodrigo Della Méa Plentz rodrigop@ufcspa.edu.br}

Fisioterapeuta. Doutor em Ciências pela UNIFESP. Professor do Departamento de Fisioterapia da Fundação Universidade Federal de Ciências da Saúde de Porto Alegre (Porto Alegre/Brasil). Currículo Lattes: http://lattes.cnpq.br/0243728836855726 


\section{CONHECIMENTO}

\section{RESUMO}

No Brasil, a Atenção Primária em Saúde (APS) é porta de entrada no serviço de saúde do Sistema Único de Saúde (SUS). A rede é constituída por unidades de saúde onde profissionais preparados para atuar na prevenção e manutenção da saúde da comunidade buscam atender às necessidades locais de forma equitativa, eficaz e precisa. O objetivo deste estudo é descrever o perfil dos profissionais da APS, vinculados a Especialização em Saúde da Família - UNA-SUS UFCSPA. Foi realizado um estudo transversal com profissionais da saúde não participantes do Programa Mais Médicos, que atuam na APS no Rio Grande do Sul vinculados ao curso, entre 2011 e 2013, para observar as características dos profissionais e o seu vínculo com o trabalho. Entre os 277 avaliados, a maioria são mulheres, enfermeiras, com média de 35 anos, formadas a menos de 10 anos, 88\% trabalham em ESF, e 73,1\% participam de equipes de saúde bucal. A pós-graduação concluída direcionada para APS foi observada em $30 \%$ dos profissionais; e 56,3\% estavam no primeiro trabalho em APS, com media de 4,5 anos de atuação; 40,4\% tinham outra atividade remunerada (77\% médicos). Observou-se que $59 \%$ estavam satisfeitos com o tipo de vínculo, e entre a maioria foi observada a satisfação com as condições gerais de trabalho $(58,1 \%)$, com a estrutura física e com os equipamentos das unidades (58,3\%). Observaram-se profissionais com menor idade e pouca especialidade na área, entretanto, todos eles buscando aprimoramento através da Especialização para melhorar sua atuação na APS.

Palavras-chave: Atenção Primária à Saúde. Saúde da Família. Profissionais da Saúde.

\section{ABSTRACT}

In Brazil Primary Health Care (PHC) is the gateway to health service of the Unified Health System. The network consists of health unit where professionals seek to meet local needs fairly and effectively, and it must rely on trained professionals to work in community health prevention and maintenance. The aim of this study is to describe the profile of PHC professionals who are linked to UNA-SUS-UFCSPA's Specialization Program in Family Health. A cross-sectional study with health professionals who work in PHC in Rio Grande do Sul and were linked to the program between 2011 and 2013 was conducted to observe the characteristics of professionals and their relationship with work. Among the participants (277), the majority are women, nurses with an average of 35 years, professionals for less than 10 years, $88 \%$ work in FHS, and $73.1 \%$ with oral health team. The completed a graduate degree focused on PHC was observed in $30 \%$ of the professionals; $56.3 \%$ were working their first job in PHC, with a median of 4.5 years of operation; and $40.4 \%$ engaged in another paid activity (77\% physicians). It was observed that $59 \%$ were satisfied with their type of employment, and most participants showed to be satisfied with their general working conditions (58.1\%) and the physical structure and equipment of the health unit (58.3\%). Younger professionals and with little expertise in the area were observed, however all of them were seeking development through Specialization to improve their performance in PHC.

Keywords: Primary Health Care. Family Health. Health Personnel. 


\section{CONHECIMENTO}

\section{INTRODUÇÃo}

Por todo o mundo os sistemas de saúde vêm sofrendo profundas mudanças devido às transições demográficas, epidemiológicas e até mesmo pela carga de incapacidade da estrutura. Estas transições são poderosas forças que provocam mudanças nos sistemas de atendimento e também nas características dos profissionais de saúde (CRISP e CHEN, 2013).

A Atenção Primária à Saúde (APS) vem sendo amplamente utilizada como estratégia para orientar a organização do sistema de saúde e responder as necessidades da população. Sua boa organização é apontada como contribuinte para a melhora dos cuidados e têm impactos positivos na saúde da população e na eficiência do sistema de atenção (GIOVANELLA et al, 2009). No Brasil a APS (também conhecida por Atenção Básica à Saúde) tem sido utilizada como estratégia para organizar os serviços e promover a equidade em saúde e, através da Estratégia Saúde da Família (ESF), tem sido apontada como prioritária para a reorientação do modelo de atenção no Sistema Único de Saúde (SUS) (ARAÚJO et al, 2014) e sua importância reafirmada pela atual Política Nacional da Atenção Básica 2017 (BRASIL, 2017).

Com a mudança do modelo de atenção do SUS associada à implantação da ESF, surgiu a necessidade de formar um profissional preparado para atuar na APS, o profissional éessencialmente parte do complexo processo de atenção à saúde, conjunto que resulta na assistência a população (PIRES, 2008; FERTONANI, 2015). Essa mudança no processo de trabalho da equipe de saúde deve deixar de focar apenas na atenção às doenças e ter seu foco dirigido à promoção e manutenção da saúde. Para sua consolidação é necessário que se mantenha a aquisição constante de conhecimentos técnicos-científicos; mas também é imperativa a substituição da visão curativa pela visão prognóstica. Assim, como resultante, pode-se potencializar a capacidade de produzir resultados positivos que tenham impacto sobre os principais indicadores de saúde e qualidade de vida da população (CONASS, 2007). Corroborando com a importância da qualificação e a formação de seus profissionais, tem-se potencializado nos últimos anos a oferta de cursos para os profissionais da saúde, principalmente aos que já estão atuando nos serviços de saúde (MATTOS et al, 2015).

A interdisciplinaridade é premissa do trabalho em equipe da ESF e a equipe multiprofissional mínima é composta pelo médico, enfermeiro, auxiliar ou técnico de enfermagem e os agentes comunitários de saúde, e pode ainda conter a equipe de saúde bucal composta por cirurgião-dentista e auxiliar e/ou técnico em Saúde Bucal (BRASIL, 2017). Destaca-se que os profissionais da saúde e suas competências juntamente com os recursos estruturais, são parte importante do processo de atenção aos usuários do sistema (DONABEDIAN, 2005). Assim, ele deve ter qualificação e perfil diferenciado, trabalhar como equipe multiprofissional e ser capaz de desenvolver e organizar ações que enfatizem a relação entre 


\section{CONHECIMENTO}

a equipe, a comunidade e os diversos setores envolvidos na promoção da saúde (COTTA et al, 2006). Embora ainda existam obstáculos, observa-se que um melhor resultado dos atributos da APS está relacionado com a melhora qualificação dos seus profissionais (OLIVEIRA et al, 2016).

A grande relevância do serviço de APS que é fornecido para a população brasileira, demonstra a fundamental importância de se priorizar também a qualificação das equipes (FELISBERTO, 2004; BRASIL, 2017).

Consoante a esta necessidade, o Ministério da Saúde criou em 2010 a Universidade Aberta do SUS (UNA-SUS) para atender às necessidades de formação e educação permanente, visando à resolução de problemas presentes no cotidiano dos profissionais de saúde que atuam no SUS. Formada por uma rede colaborativa composta em 2015 por 35 instituições de ensino superior do Brasil oferece todos os cursos gratuitos e na modalidade de educação à distância (EAD) para facilitar o acesso ao conteúdo, que possuem diversos níveis de capacitação acadêmica (BRASIL, 2015).

Naárea da saúde, a avaliação tem como propósito fundamental dar suporte aos processos decisórios no âmbito do sistema de saúde, possibilitando a identificação de problemas e a reorientação das ações e dos serviços executados (BRASIL, 2009). A nova administração pública vem se consolidando no Brasil e, com isso, se amplia a importância da avaliação, servindo de instrumento indispensável para observação e controle por parte das instituições do Estado (CARNUT e NARVAl, 2016).

Tendo em vista a importância de se conhecer melhor o perfil dos profissionais que trabalham na rede e que participam do processo de atenção à saúde da população brasileira, é objetivo desse estudo descrever o perfil dos profissionais da Atenção Primária à Saúde que participaram da Especialização em Saúde da Família, através da Universidade Aberta do Sistema Único de Saúde (UNA-SUS).

\section{MÉTODO}

Trata-se de um estudo transversal e descritivo, com a população composta por 473 alunos matriculados no Curso de Especialização em Saúde da Família - UNA-SUS - UFCSPA (Universidade Federal de Ciências da Saúde de Porto Alegre) que participaram do curso entre março de 2011 e março de 2013. Essa população é composta por médicos, dentistas e enfermeiros que atuam nas unidades de saúde de APS do estado do Rio Grande do Sul, e não estavam vinculados ao "Programa Mais Médicos". As informações deste grupo foram obtidas através do banco de dados de registro dos alunos da UNA-SUS UFCSPA. Todos os profissionais vinculados ao Curso foram convidados a fazer parte da pesquisa, sendo realizada a avaliação com aqueles que concordaram em participar do estudo. 


\section{CONHECIMENTO}

Foram excluídos os alunos que não estavam em dia com as atividades do Curso, aqueles que estavam afastados do atendimento na Unidade de Saúde no momento da coleta, os alunos evadidos e aqueles que apresentaram discordância nas informações na realização do controle de qualidade dos dados da pesquisa (Figura1, ao fim do trabalho).

0 instrumento de coleta foi aplicado através de questionário estruturado em plataforma web com questões referentes a informações sócio demográficas, educacionais, capacitação profissional, sobre a equipe e o processo de trabalho.

As coletas ocorreram entre agosto de 2012 e julho de 2013, iniciado com um primeiro contato realizado através de e-mail com convite para participação na pesquisa. Juntamente com o e-mail foram enviadas as informações sobre a pesquisa e o link para acesso ao questionário on-line com orientações. Para os casos em que não se obteve resposta ao e-mail, foram realizadas até 5 novas tentativas de contato, preferencialmente via telefone e e-mail, convidando para participação na pesquisa.

A entrada de dados foi realizada via formulário on-line, uma vez que foi coletado através de questionário digital, disponibilizado no servidor "Survey Monkey" (www.surveymonkey.com) e exportados para plataforma estatística SPSS (IBM - Statistical Product and Service Solutions).

Para controle de qualidade dos dados foram selecionados por sorteio $10 \%$ dos entrevistados, para responder via telefone alguns itens dos instrumentos anteriormente aplicados, as perguntas foram selecionadas aleatoriamente em conglomerados para todos os selecionados. A coleta do controle de qualidade foi realizada entre 30 e 60 dias após a primeira coleta, e os dados foram confrontados com os dados completos. Em caso de divergência, o profissional e os dados da entrevista foram excluídos da pesquisa.

Os resultados são apresentados através de percentuais e as variáveis quantitativas também são demonstradas por meio de suas medidas de tendência central e dispersão. Eventuais não respostas foram consideradas como inexistentes (missing), não sendo incluídas nos cálculos percentuais.

Para as análises comparativas foram utilizados os testes ANOVA one-way para variáveis com distribuição simétrica, e teste de Kurskal Wallis para variáveis com distribuição assimétrica. 0 nível de significância empregado foi de 5\% para avaliação bicaudal em todos os testes estatísticos. Para a análise estatística foi utilizado o pacote estatístico SPSS (versão 23).

Todos os entrevistados confirmaram sua participação através do Termo de Consentimento Livre e Esclarecido. Este projeto foi aprovado pelo Comitê de Ética em Pesquisa da Universidade Federal de Ciências da Saúde de Porto Alegre (UFCSPA) sob parecer nº 1333/11. 


\section{CONHECIMENTO}

\section{Regultados}

No período do estudo foram elegiveis 473 profissionais do Curso de Especialização em Saúde da Família (UNA-SUS - UFCSPA) que atuavam na APS no Rio Grande do Sul. Após todos os alunos regulares terem sido convidados para participar do censo, 277 alunos que se enquadravam nos critérios de elegibilidade, responderam a solicitação e concordaram em participar da pesquisa, alcançou-se uma taxa de resposta de $58,56 \%$.

Observou-se entre os alunos do curso (473) a maioria com graduação em enfermagem (60\%). Entre todos os profissionais, $29,1 \%$ já possuíam pós-graduação (residência, especialização ou mestrado), mas somente 8,6\% com pós-graduação em áreas afins à APS (Tabela 1). Foram considerados como cursos de pós-graduação na área da APS os diversos programas em saúde coletiva, saúde pública, saúde da família, atenção básica e medicina da família e comunidade e excluídos deste grupo os cursos voltados para gestão dos serviços.

Para o grupo de entrevistados (277) também se verificou maior número de profissionais da enfermagem $(64,3 \%)$ e menor número de médicos respondentes $(9,4 \%)$, e observou-se que $57,4 \%$ dos profissionais já possuíam alguma pós-graduação concluída, sendo $30 \%$ na área da APS (Tabela 1).

0 gênero feminino foi o de maior número entre os profissionais, independentemente de formação, tanto entre os alunos do curso $(77,5 \%)$ quanto nos alunos entrevistados $(84,5 \%)$, com o gênero masculino mais frequente somente entre o os médicos do Curso.

Tabela 1: Caracterização dos alunos matriculados na Especialização UNA-SUS UFCSPA (2012-2014) e dos alunos entrevistados na pesquisa.

\begin{tabular}{lll}
\hline Variável & $\begin{array}{l}\text { População do curso } \\
(\mathbf{N = 4 7 3 )}\end{array}$ & $\begin{array}{l}\text { Entrevistados } \\
(\mathbf{n = 2 7 7 )}\end{array}$ \\
\hline $\begin{array}{l}\text { GRADUAÇÃO (\%) } \\
\text { Medicina }\end{array}$ & \\
Enfermagem & 17,3 & 9,4 \\
Odontologia & 60,0 & 64,3 \\
\hline PóS-GRADUAÇÃo coNCLUÍDA (\%) & 22,7 & 26,4 \\
Não* & & \\
Sim (inclui residência)* & $\mathbf{7 0 , 9}$ & $\mathbf{4 2 , 6}$ \\
Médico & $\mathbf{2 9 , 1}$ & $\mathbf{5 7 , 4}$ \\
Enfermeiro & 23,1 & 42,3 \\
Dentista & 32,0 & 64,0 \\
Na área de APS * & 26,0 & 46,6 \\
\hline
\end{tabular}




\begin{tabular}{|c|c|c|c|}
\hline \multicolumn{4}{|c|}{ GENERO /FORMAÇÃO (\%) } \\
\hline \multirow[t]{2}{*}{ Medico } & Feminino & 47,0 & 61,5 \\
\hline & & 53,0 & 38,5 \\
\hline \multicolumn{2}{|c|}{ Enfermeiro Feminino } & $\begin{array}{l}91,4 \\
86\end{array}$ & 93,8 \\
\hline \multirow[t]{2}{*}{ Dentista } & Feminino & 64,3 & 69,9 \\
\hline & Masculino & 35,7 & 30,1 \\
\hline \multicolumn{4}{|c|}{ FAIXAS ETÁRIAS (\%) } \\
\hline \multirow[t]{3}{*}{ Medico } & $<30$ & 23,9 & 7,7 \\
\hline & 30 a 44 & 59,0 & 69,2 \\
\hline & $\geq 45$ & 17,1 & 23,1 \\
\hline & 28,1 & 29,8 \\
\hline \multicolumn{2}{|c|}{$\begin{array}{r}\text { Enfermeiro }<30 \\
30 \text { a } 44\end{array}$} & 61,7 & 60,1 \\
\hline & $\geq 45$ & 10,3 & 10,1 \\
\hline \multirow{3}{*}{ Dentista } & $<30$ & 30,5 & 20,5 \\
\hline & 30 a 44 & 54,5 & 60,3 \\
\hline & $\geq 45$ & 14,9 & 19,2 \\
\hline \multicolumn{4}{|c|}{ SITUAÇÃO CONJUGAL (\%) } \\
\hline \multicolumn{2}{|c|}{ Em união estável } & 32,8 & 56,3 \\
\hline \multicolumn{2}{|c|}{ Sem união estável } & 67,2 & 43,7 \\
\hline \multicolumn{2}{|c|}{ IDADE (Média $\pm D P$ )** } & $34,89( \pm 08,02)$ & $35,95( \pm 07,95)$ \\
\hline \multicolumn{2}{|c|}{ Medico } & $37,05( \pm 10,21)$ & $39,19( \pm 08,98)$ \\
\hline \multicolumn{2}{|c|}{ Enfermeiro } & $34,14( \pm 07,15)^{* *}$ & $33,99( \pm 06,89)^{* *}$ \\
\hline \multicolumn{2}{|c|}{ Dentista } & $35,21( \pm 08,01)$ & $36,77( \pm 08,14)$ \\
\hline \multicolumn{2}{|c|}{ TEMPO DE FORMADO (Média $\pm D P$ )* } & $9,95(6,78)$ & $10,66(7,20)$ \\
\hline \multicolumn{2}{|c|}{ Médico } & $11,1( \pm 9,82)$ & $12,11(9,11)$ \\
\hline \multicolumn{2}{|c|}{ Enfermeiro } & $8,88( \pm 5,52)$ & $8,56(5,20)$ \\
\hline \multicolumn{2}{|c|}{ Dentista } & $11,49( \pm 7,46)$ & $10,66(7,20)$ \\
\hline \multicolumn{2}{|c|}{ TEMPO DE FORMADO (Mediana - Q25\%-75\%)* } & $8(5-12)$ & $8(5-13)$ \\
\hline \multicolumn{2}{|c|}{ Médico } & $7(4-14,5)$ & $10,5(4-18)$ \\
\hline \multicolumn{2}{|c|}{ Enfermeiro } & $7(5-11)$ & $7(5-11)^{* * *}$ \\
\hline \multicolumn{2}{|c|}{ Dentista } & $9(6-16)^{* * *}$ & $9,5(5-18)$ \\
\hline
\end{tabular}

*referente ao total de profissionais do grupo; ${ }^{* *} \mathrm{p}<0,001$ para comparação entre idade de médicos e enfermeiros; *** $p<0,05$ teste de Kurskal Wallis (entre profissionais, intra-grupo); Fonte: Os dados da população do curso foram retirados do banco de dados UNA-SUS UFCSPA; os dados dos entrevistados são parte dos resultados da pesquisa.

A faixa etária (dividida em três grupos para avaliar profissionais no início, intermédio e a com mais tempo na profissão) teve predomínio para todos os grupos de profissionais entre 30 e 44 anos. As 
médias de idade apresentaram comportamento semelhante entre os grupos, entretanto os enfermeiros apresentaram menor média de idade que os demais, com diferença estatisticamente significativa $(p<0,001)$ quando comparada com médicos. A situação conjugal "em união estável" foi mais frequente no grupo entrevistado $(56,1 \%)$ do que na população do estudo $(32,8 \%)$ (Tabela 1).

Ao observar o tempo de formado, verificou-se uma distribuição assimétrica, sendo observada mediana de 8 anos, em ambos os grupos (população do curso e entrevistados). Entretanto, verifica-se diferença estatisticamente significativa $(p<0,01)$ entre as profissões, com menor tempo de formado dos profissionais enfermeiros (Tabela 1).

Outro grupo de variáveis foi verificado através do instrumento da pesquisa que foi disponibilizado para o grupo entrevistado. As características relacionadas ao trabalho foram avaliadas, onde se pode verificar a maioria dos profissionais $(88,4 \%)$ alocados em Unidades de Saúde da Família, 72,9\% com equipes de saúde bucal (Tabela 2).

Tabela 2: Características relativas ao trabalho dos profissionais entrevistados.

\begin{tabular}{|c|c|c|c|}
\hline Variável & $\begin{array}{l}\text { Percentual } \\
(\%)\end{array}$ & $\begin{array}{l}\text { Frequência } \\
\text { (n) }\end{array}$ & $\begin{array}{l}\text { Média ( } \pm \text { DP) ou } \\
\text { Mediana (Q25-75\%) }\end{array}$ \\
\hline \multicolumn{4}{|l|}{ Modalidade de atenção da UBS } \\
\hline Saúde da Família & 88,4 & 245 & \\
\hline Tradicional & 10,8 & 30 & \\
\hline Parametrizada & 0,8 & 2 & \\
\hline \multicolumn{4}{|l|}{ Possui equipe de saúde bucal na unidade } \\
\hline $\operatorname{Sim}$ & 72,9 & 202 & \\
\hline Primeiro trabalho em Equipe de SF & $56,3^{*}$ & 156 & \\
\hline Médicos & 46,2 & 12 & \\
\hline Enfermeiros & 56,2 & 100 & \\
\hline Dentistas & 68,5 & 50 & \\
\hline Tempo de atuação em APS & & & $4,5(2,67-8,17)^{*}$ \\
\hline Médico & & & $4,50(2,75-8,5)$ \\
\hline Enfermeiro & & & $4,58(2,5-7,54)$ \\
\hline Dentista & & & $4,42(3,17-9,0)$ \\
\hline Tempo de atuação nesta equipe SF (anos) & & & $2,5(1,1-4,58)^{*}$ \\
\hline Médicos & & & $4,03(2,18-7,47)$ \\
\hline Enfermeiros & & & $2,33(1,08-5,00)^{* * *}$ \\
\hline Dentistas & & & $4,36(1,42-8,18)$ \\
\hline
\end{tabular}


Carga horária semanal de trabalho na equipe

Médicos

Enfermeiros

Dentistas
$38,9( \pm 4,62)^{*}$

$38,2( \pm 5,51)$

$39,7( \pm 2,47)$

$37,2( \pm 7,11)$

\section{Tipo de seleção para contrato}

Concurso

Processo seletivo

Indicação

Entrevista

Outros

$\begin{array}{ll}56,7 & 157 \\ 20,2 & 56 \\ 8,3 & 23 \\ 9,4 & 26 \\ 5,4 & 15\end{array}$

\section{TIPO DE VÍNCULO EMPREGATÍCIO}

\section{Médicos}

Concursado (Estatutário)

Funcionário público CLT

Outro

$\begin{array}{ll}34,6 & 9 \\ 19,2 & 5 \\ 46,2 & 12\end{array}$

\section{Enfermeiros}

Concursado (Estatutário)

Funcionário público CLT

Outro

\begin{tabular}{ll}
48,9 & 87 \\
27,0 & 48 \\
24,2 & 42 \\
\hline
\end{tabular}

\section{Dentistas}

Concursado (Estatutário)

Funcionário público CLT

Outro

Inserido em Plano de Carreira

Médicos

Enfermeiros

Dentistas

$48 \quad 35$

$26 \quad 19$

$26 \quad 19$

$20,2^{*} \quad 56$

$15,4 \quad 4$

$22,5 \quad 40$

$17,8 \quad 13$

\section{Satisfação com o tipo de vínculo:}

\begin{tabular}{lll} 
Insatisfeito (e totalmente insatisfeito) & 34,7 & 96 \\
Indiferente & 6,5 & 18 \\
Satisfeito (e totalmente satisfeito) & 58,8 & 163 \\
\hline Outra atividade remunerada & $\mathbf{4 0 , 4 ^ { * }}$ & $\mathbf{1 1 2}$ \\
Médicos & 76,9 & 20 \\
Enfermeiros & 25,3 & 45 \\
Dentistas & 64,4 & 47
\end{tabular}




\begin{tabular}{lll}
$\begin{array}{l}\text { Como você classifica as condições gerais de } \\
\text { trabalho na sua Equipe de Saúde da Família? }\end{array}$ & & \\
Médicos & & \\
Insatisfeito (e totalmente insatisfeito) & 38,5 & 10 \\
$\quad$ Indiferente & 7,7 & 2 \\
$\quad$ Satisfeito (e totalmente satisfeito) & 53,8 & 14 \\
\hline Enfermeiros & & \\
Insatisfeito (e totalmente insatisfeito) & 39,2 & 70 \\
$\quad$ Indiferente & 3,3 & 5 \\
Satisfeito (e totalmente satisfeito) & 57,5 & 102 \\
\hline Dentistas & & \\
Insatisfeito (e totalmente insatisfeito) & 29,9 & 22 \\
Indiferente & 9 & 6 \\
Satisfeito (e totalmente satisfeito) & 61,1 & 45 \\
\hline
\end{tabular}

Frequência de reunião entre os membros da equipe

$\begin{array}{lll}\text { Raramente } & 7,6 & 21 \\ \text { Trimestralmente } & 2,5 & 7 \\ \text { Mensalmente } & 13,0 & 36 \\ \text { Quinzenalmente } & 11,2 & 31 \\ \text { Semanalmente } & 65,7 & 182\end{array}$

Curso de especialização pode provocar mu- 95,7* 265

danças na atuação profissional

$\begin{array}{lll}\text { No seu dia-a-dia de trabalho } & 38,3 & 106 \\ \text { Na sua visão sobre a assistência à saúde } & 20,9 & 58 \\ \text { No gerenciamento dos serviços e ações } & 11,2 & 31 \\ \text { Na tomada de decisões } & 9,7 & 27 \\ \text { Nas ações estratégicas } & 8,3 & 23 \\ \text { Na sua relação com a comunidade } & 7,2 & 20 \\ \text { Nas relações interinstitucionais } & 4,3 & 12\end{array}$

*refere-se ao total de profissionais (não divididos por graduação); ** - Incluídos como outro vínculo empregatício: cooperativado, temporário, contrato de prestação de serviço, por organização de Saúde, organização da sociedade civil de interesse público, sem vínculo (RPA), etc); **p<0,05 teste de Kurskal Wallis (entre profissionais).

Constatou-se que $56,3 \%$ dos entrevistados estavam em seu primeiro trabalho em uma ESF, sendo menos frequente entre os médicos $(46,2 \%)$ e maior entre os dentistas $(68,5 \%)$. 0 tempo de atuação em APS foi de aproximadamente 4,5 anos e a carga horária semanal de trabalho próxima de 38 horas/ semanais e não houve diferenças significativas entre os grupos profissionais. Já o tempo de atuação na 


\section{CONHECIMENTO}

ESF (no momento da entrevista) teve variação importante entre os profissionais, sendo maior entre os dentistas e médicos, e estatisticamente menor entre os profissionais de enfermagem (Tabela 2).

0 processo de contratação mais frequente foi através de concursos $(56,7 \%$ ), com $20,2 \%$ dos profissionais inseridos em plano de carreira sendo mais frequente entre os enfermeiros $(22,5 \%)$ que para os demais profissionais.

Observou-se ainda o tipo de vínculo do profissional, e constatou-se que os médicos apresentaram menor quantidade de vínculo como estatutário e como funcionário público (CLT) quando comparados com os demais profissionais, e apresentam maior percentual de contratados pelo terceiro setor e por vínculos temporários (Tabela 2).

Ainda verificou-se que os profissionais que tem plano de carreira são na maioria $(87,8 \%)$ contratados pelo regime estatutário ou funcionário público. Entretanto, somente $38,1 \%$ dos estatutários e 6,5\% dos funcionários públicos (CLT) informaram estar inseridos em plano de carreira, o que indica não haver relação direta do tipo de vínculo.

A satisfação com o tipo de vínculo empregatício alcança 58,8\% dos profissionais, e a presença de outra atividade remunerada simultânea ao trabalho na APS é verificada em 40,4\% dos profissionais, destacando-se maiores percentuais entre os médicos e dentistas.

Entre os satisfeitos com o vínculo, destacam-se os estatutários (78,7\% satisfeitos e totalmente satisfeitos), e funcionários públicos (63\% satisfeitos e totalmente satisfeitos). Os maiores índices de insatisfação (insatisfeito e totalmente insatisfeito) foram encontrados entre os temporários $(66,7 \%)$ e contratados $(66,7 \%)$. Entre os diferentes profissionais, observou-se que a insatisfação com o tipo de vínculo mais elevada entre médicos $(47,6 \%)$, seguido pelos dentistas $(32,8 \%)$ e depois os enfermeiros $(27,6 \%)$.

Também foi avaliada a satisfação do profissional com as condições gerais de trabalho na equipe, percebe-se uma distribuição heterogênea com maior presença de profissionais satisfeitos $(58,1 \%)$, mais frequente entre os dentistas (53,8\%). A insatisfação com as condições gerais foi apontada por $39,2 \%$ dos enfermeiros, $38,5 \%$ dos médicos e $29,9 \%$ dos dentistas (Tabela 2 ).

Grande parcela das equipes dos profissionais avaliados realiza reuniões internas semanalmente ou quinzenalmente $(76,9 \%)$, o que pode refletir positivamente no planejamento das ações de atenção à saúde da população (Tabela 2).

A realização da Especialização em Saúde da Família foi apontada como incentivador de mudanças para a grande maioria dos profissionais $(95,7 \%)$, e quando questionados sobre as possíveis mudanças provocadas pela especialização em questão, foram mais citadas mudanças "no seu dia-a-dia de trabalho" (38,3\%) e "na visão sobre a assistência à saúde" (20,9\%), entre outras (Tabela 2). 
Outro ponto de interesse desta pesquisa foi avaliar algumas características relacionadas à educação permanente, e mesmo sendo observado que $87,4 \%$ tinham acesso a materiais de atualização (impresso ou on-line) na área da APS e que $44,8 \%$ dos profissionais participaram de algum tipo de treinamento introdutório em APS (destacando a maior participação dos enfermeiros), ainda assim a necessidade de educação permanente foi apontada por 98,5\% dos profissionais (Tabela 3).

Tabela 3: Características ligadas ao processo de educação permanente dos entrevistados.

\begin{tabular}{|c|c|c|}
\hline Variável & $\begin{array}{l}\text { Percentual } \\
(\%)\end{array}$ & $\begin{array}{l}\text { Média ( }(\mathrm{DP}) \text { ou } \\
\text { Mediana (Q25\% - 75\%) }\end{array}$ \\
\hline $\begin{array}{l}\text { Participou de treinamento/capacitação introdutória } \\
\text { em Atenção Primária em Saúde. }\end{array}$ & 44,8 & \\
\hline Médico & 34,6 & \\
\hline Enfermeiro & 50,0 & \\
\hline Dentista & 35,6 & \\
\hline $\begin{array}{l}\text { Sente necessidade de Educação Permanente em } \\
\text { Saúde da Família. }\end{array}$ & 98,5 & \\
\hline Médico & 96,2 & \\
\hline Enfermeiro & 98,9 & \\
\hline Dentista & 97,3 & \\
\hline $\begin{array}{l}\text { Tem acesso à material de atualização na área de } \\
\text { Atenção Primária à Saúde. }\end{array}$ & 87,4 & \\
\hline Médico & 84,6 & \\
\hline Enfermeiro & 91,6 & \\
\hline Dentista & 78,1 & \\
\hline
\end{tabular}

\begin{tabular}{|c|c|c|}
\hline \multicolumn{3}{|c|}{$\begin{array}{l}\text { Estimulado a participar do curso de Especialização em Saúde da } \\
\text { Família por: }\end{array}$} \\
\hline Gestor & 6,9 & \\
\hline Decisão pessoal & 76,3 & \\
\hline Colegas & 14,6 & \\
\hline Outros & 2,2 & \\
\hline $\begin{array}{l}\text { Tem horas previstas de atividades de educação per- } \\
\text { manente. }\end{array}$ & $34,4^{*}$ & $8(4-12)^{*}$ \\
\hline Médico & 14,3 & $4(2-* *)$ \\
\hline Enfermeiro & 34,0 & $8(4-12)$ \\
\hline Dentista & 41,8 & $8(4-11,25)$ \\
\hline
\end{tabular}




\section{Considerando a infraestrutura física e equipamentos, a unidade de saúde está adequada para o desenvolvimento das ações básicas de saúde e 0 acolhimento dos usuários.}

$\begin{array}{ll}\text { Muito Insatisfatório } & 12,8 \\ \text { Insatisfatório } & 18,9 \\ \text { Regular } & 19,6 \\ \text { Satisfatório } & 24,5 \\ \text { Muito Satisfatório } & 14,2\end{array}$

*refere-se ao total de profissionais (não divididos por graduação); ${ }^{* *}$ valor inexistente ( ${ }^{\circ}$. insuficiente).

A maior parte dos profissionais ingressou por decisão pessoal $(76,3 \%)$ e somente $6,9 \%$ foram estimulados pela gestão/gestor do serviço. Embora tenha se observado que $34,4 \%$ dos profissionais têm prevista carga horária para educação permanente, somente $58 \%$ deles indicaram o número de horas reservadas para a atividade, sendo apresentada pelos enfermeiros e dentistas, com períodos semelhantes (Tabela 3).

Os profissionais foram questionados sobre a percepção da infraestrutura física e equipamentos da unidade, e verificou-se $30,7 \%$ dos profissionais satisfeitos, ampliando para condições no mínimo regulares encontra-se $58,3 \%$ dos profissionais (Tabela 3 ).

\section{Discussão}

Nesse estudo verificou-se que a maioria dos profissionais da APS que realizaram o curso no período estudado eram mulheres, maior frequência de profissionais da enfermagem, e entre a faixa etária dos 30 a 44 anos. 0 tempo de formado dos profissionais ficou próximo aos 8 anos, tendo sua atuação em APS com mediana de 4,5 anos. Salienta-se a presença de outra atividade remunerada entre os médicos e dentistas e a distribuição heterogênea na satisfação com as condições de trabalho na equipe.

A avaliação foi realizada através de pesquisa on-line, que é cada vez mais popular e apresenta diversas vantagens aos pesquisadores e ao pesquisado, pois propicia benefícios como um maior conforto e praticidade, economiza tempo, e tem maior facilidade de responder. Nesta pesquisa alcançou-se uma participação de $58,56 \%$ dos convidados, uma taxa superior ao esperado para esta forma de coleta de pesquisa. A não resposta é um problema comum em levantamentos de larga escala, e para minimizar esse viés, foram utilizadas algumas técnicas como o contato telefônico efetivo além do e-mail, realizada em até 5 tentativas efetivadas, salientando aos participantes a importância de responder aos instrumentos da pesquisa, e esse procedimento parece ter sido determinante na maior taxa de resposta. 


\section{CONHECIMENTO}

Observou-se que as características dos pesquisados apresentam distribuição semelhante às características dos profissionais dasaúde no âmbito geral no mesmo período do estudo, que demonstraram uma feminização (em nosso estudo, foi menor na classe médica). Também houve predominância na faixa da meia idade (em nosso estudo: 30-44 anos) inclusive nas equipes de Saúde da Família onde os profissionais enfermeiros também são em maior número. Estes achados vão de encontro com outros estudos sobre os profissionais da saúde no Brasil (CARRILLO-GARCÍA et al., 2013; COSTA et al., 2013; TOMAZI et al., 2008; PINTO et al., 2010; FIUZA et al., 2012; OLIVEIRA et al., 2016).

Observou-se parcela maior de profissionais "sem união estável", o que pode estar associada às idades mais jovens, e também à mudança de hábitos matrimoniais no país (PINTO et al., 2010; PLATONOW, 2013), mas se verificou maior participação na pesquisa de profissionais "em união estável". O comportamento da idade e o tempo de formado entre os diferentes profissionais e entre os grupos foram semelhantes neste estudo.

A modalidade de Saúde da Família foi mais frequente entre os entrevistados, sendo que grande parcela também possui equipe de saúde bucal. Facchini et al (2006) em um estudo realizado em diferentes regiões do Brasil, apontou que a oferta de ações, a sua utilização e o contato por ações programáticas foram mais adequados na ESF embora o desempenho da APS ainda estivesse distante das pretensões do SUS. Esse resultado é reafirmado por Castro et al (2012) que observaram que, de acordo com a experiência dos profissionais, a ESF, em comparação à Unidade Básica de Saúde (UBS), apresenta significativamente maior grau de orientação à APS.

Verificou-se nesta pesquisa que $56,3 \%$ dos profissionais estavam trabalhando pela primeira vez em Equipe de Saúde da Família, e que apresentavam tempo mediano próximo de 4,5 anos tanto para o tempo de atuação na atual equipe como tempo de atuação na área de APS.

A presença de profissionais mais jovens atuando na ESF pode ser um reflexo das mudanças curriculares nos cursos de graduação, onde tem sido enfatizada a capacitação profissional para as reais demandas da APS (COSTA et al., 2013). De encontro, as instâncias superiores começaram incrementar ações e programas no sistema educacional e criando incentivos para a reorientação da formação, como as Diretrizes Curriculares (DCN), o Sistema de Avaliação da Educação Superior (Sinaes) O Programa de Incentivo às Mudanças Curriculares das Escolas Médicas (Promed), o Pró-Saúde, o PET-Saúde, Programa de Formação de Nivel Médio para a Saúde (PROFAPS), entre outros (AUTONOMO, 2015), buscando estimular os novos profissionais a trabalhar na Atenção Básica do SUS e também, de acordo com a Política Nacional de Atenção Básica (PNAB), desenvolver mecanismos para sua qualificação (BRASIL, 2017), com a criação do PROVAB, do "Programa Mais Médicos", da Universidade Aberta do SUS (UNASUS), entre outros. 


\section{CONHECIMENTO}

O comportamento da carga horária de trabalho vai de encontro com a legislação para os profissionais daESF, que de acordo com a Portaria do Ministério da Saúde N².488 (21 de outubro de 2011) determina o regime de 40 horas para todos os profissionais, com exceção dos médicos. Ela também prevê a dedicação mínima de 32 horas para atividades na ESF, podendo as demais ser prestadas em outras atividades de serviço ou para educação permanente.

Os processos de seleção dos profissionais podem ser definidos de acordo com o interesse da gestão local, e nesta pesquisa observou-se um grande número de vínculos realizados através de concurso ou processo de seleção.

A satisfação com as condições de trabalho na equipe reflete no engajamento do profissional no serviço, por isso deve ser visto como importante ponto na qualidade das ações, uma vez que o processo do trabalho em equipe pode refletir no serviço prestado.

A formação e a qualificação para APS é uma estratégia para garantir a universalidade e integralidade do sistema de saúde, pois através dela pode-se criar um espaço de construção coletiva entre os diversos sujeitos envolvidos no cuidado em saúde (D'AVILA et al., 2014).

A educação em saúde no contexto dos serviços pode ser abordada através de políticas norteadoras dos processos educativos contínuos, com suas diversas denominações (capacitações, treinamentos, cursos, atualizações, entre outros) (MIRANDA et al., 2008), e deve ser encarada como uma importante "estratégia de gestão", com grande potencial provocador de mudanças no cotidiano dos serviços. Desta forma, ela torna-se uma oportunidade de ampliação e valorização dos saberes individuais e coletivos envolvidos no processo de cuidar da saúde e melhoria da qualidade da assistência (WEIKAMP et al., 2016).

Embora entre os profissionais avaliados nesta pesquisa poucos apresentem pós-graduação voltada para a APS, todos estavam cursando uma Especialização em Saúde da Família, reconhecimento da importância e a necessidade de aprimoramento específico para melhorar a atuação profissional na área. Também é necessário salientar o tempo de atuação na APS (mediana de 4,5 anos), tempo relativamente curto para que ocorra a sensibilização e realização de aprimoramento na área. Corroborando com nosso estudo, Oliveira et al (2016) verificaram que o tempo médio de trabalho na equipe de ESF foi de 3,4 anos, sendo menor para os profissionais médicos, com média de 1,35 anos na mesma equipe. Outra pesquisa realizada em Montes Claros descreve alto índice de rotatividade dos profissionais das ESFs, sendo que os médicos permanecem por menos tempo (média de 14,5 meses), e os profissionais de nivel técnico com maior tempo de permanência (TONELLI et al., 2018). Em diversas capitais pesquisadas, as condições de trabalho, a exigência da carga horária integral, a baixa oportunidade de progressão profissional são os principais motivos para a baixa fixação dos profissionais da atenção básica (CARVALHO, 2013). 


\section{CONHECIMENTO}

É importante destacar o planejamento educativo ascendente que identifica os problemas a serem enfrentados a partir da análise coletiva dos processos de trabalho, possibilitando a construção de estratégias que promovam o diálogo entre as políticas gerais e a singularidade dos lugares e das pessoas. Por isso é essencial que cada local proponha e desenvolva ações de educação permanente, tentando combinar características singulares com ofertas e processos mais gerais para todas as equipes do município (BRASIL, 2012).

Desta forma, com a proposta reunir os trabalhadores para um planejamento conjunto, discussão e decisão de casos e situações específicas, as reuniões de equipe podem proporcionar maior clareza sobre os papéis que cada profissional desempenha no processo de trabalho, e desta forma podem tornar-se instrutivas e construtivas no processo de planejamento das ações das equipes de saúde (BARROS, 2010). E assim contempla-se a orientação da Organização Pan-Americana da Saúde (OPAS) (MOTTA, 2001) que destaca a necessidade de melhorar o serviço prestado pelos profissionais de saúde à população, tendo como base a qualificação, capacitação e aprimoramento de seu desempenho.

Diagnosticar é fundamental, mas o mais importante vem depois, e com base no que foi avaliado, mudar a realidade, procurando o seu aperfeiçoamento (PISCO, 2006). Estudos revelam que a qualidade dos serviços de APS tem melhor resultado de acordo com a melhor qualificação dos profissionais (CASTRO et al., 2012, CHOMATAS et al., 2013).

Sabendo que os serviços bem organizados e orientados para a APS contribuem para melhorar a atenção, apresentando impactos positivos na saúde da população e também na eficiência do sistema (MACINKO et al., 2003; STARFIELD; SHI, 2002; GONTIJO et al., 2017), é importante o conhecimento das características dos profissionais, do processo de trabalho, da estrutura das unidades, e das condições de atendimento, para poder estabelecer medidas adequadas no sentido de melhorar essas situações assim como ter informações corretas para a tomada de melhores decisões (HARTZ, 2008), uma vez que as decisões para os serviços sob responsabilidade da APS variam de acordo com as características do local e da natureza dos problemas (PORTELA, 2017).

Uma informação peculiar do grupo estudado é que não foram selecionados para o estudo os profissionais participantes do Programa Mais Médicos, que pode demonstrar uma característica importante da amostra, o interesse pessoal no aprimoramento profissional, independentemente de apoio financeiro. Esta característica é apoiada pelo fato de que 75\% dos profissionais ingressaram por decisão pessoal no Curso de Especialização em Saúde da Família. Contrastando com esse fato salienta-se que os gestores da APS deveriam ser disseminadores das oportunidades e necessidades de aprimoramento dos profissionais da saúde na área, entretanto, apenas 6,9\% sentiram-se estimulados por estes. 


\section{CONHECIMENTO}

Observa-se que após a criação do SUS iniciou-se um trabalho focado na evolução do processo de atenção para APS. Para os profissionais da saúde são diversas as formas propostas para a qualificação, entre elas a possibilidade de uma educação continuada que, com o apoio de ferramentas facilitadoras como a Educação a Distância, apresenta-se como uma possibilidade de democratização do saber e do fazer para os profissionais da saúde. Mesmo com a inserção mais enfática do ensino voltado para APS nos cursos da saúde ainda existem desafios para serem vencidos que vão além da capacitação do profissional e da estrutura de saúde.

\section{CONCLUSÕES}

Buscando descrever o perfil dos profissionais da Atenção Primária à Saúde que participavam do curso de Especialização em saúde da Família da UNA-SUS, observou-se que os profissionais inseridos nas equipes de APS participantes da Especialização em Saúde da Família (UNA-SUS) são na maioria mulheres, profissionais da enfermagem, com média aproximada de 35 anos de idade e que, a maioria não possuía curso de pós-graduação, o tempo médio de formado ficou abaixo dos 10 anos. A maioria dos profissionais sentiu necessidade de educação permanente, e a opção por participar da Especialização em Saúde da Família para a maioria dos profissionais foi por decisão pessoal e não por incentivo da gestão dos serviços.

Em relação ao do trabalho, a maior parte estava vinculada a ESF, o tempo médio de atuação em Saúde da Família foi menor que 5 anos, e a presença de outra atividade remunerada foi elevada para médicos e dentistas e baixa para enfermeiros. A satisfação dos profissionais com a infraestrutura das unidades para desenvolvimento das ações básicas também aponta para a necessidade um olhar mais atento para a adequação do serviço.

\section{Órgão Financiador}

Estudo com apoio financeiro da Fundação de Amparo à Pesquisa do Estado do Rio Grande do Sul (FAPERGS) e Conselho Nacional de Desenvolvimento Científico e Tecnológico (CNPq), (Edital PRONEM/ FAPERGS/CNPq-11/2049-6), e Coordenação de Aperfeiçoamento de Pessoal de Nivel Superior (CAPES) através do projeto Pró-Ensino na Saúde (N/39). 


\section{CONHECIMENTO}

\section{REFERÊNCIAS}

ARAÚJO, L.U.A. et al. Avaliação da qualidade da atenção primária à saúde sob a perspectiva do idoso.

Ciência \& Saúde Coletiva, v. 19, n. 8, p. 3521-3532. Disponivel em: <http://www.scielo.br/scielo. php?script=sci_arttext\&pid=S1413-81232014000803521\&lng=pt>. Acesso em: 22 jan. 2015.

AUTONOMO, F. R. O. M. et al. A Preceptoria na Formação Médica e Multiprofissional com Ênfase na Atenção Primária - Análise das Publicações Brasileiras. Rev. bras. educ. med. v. 39(2): 316-327. Disponivel em: <http://www.scielo.br/scielo.php?script=sci_arttext\&pid=S010055022015000200316\&Ing=en\&nrm=iso >. Acesso em: 29 out. 2018.

BARROS, J. O. A construção de projetos terapêuticos no campo da saúde mental: apontamentos acerca das novas tecnologias de cuidado. 2010. 127 f. Dissertação (Mestrado em Ciências da Reabilitação) - Faculdade de Medicina da Universidade de São Paulo São Paulo, SP, 2010.

BRASIL - UNA-SUS. Universidade Aberta do Sistema Único de Saúde. 0 que é a UNA-SUS?. Disponível em: <http://www.unasus.gov.br/page/una-sus/o-que-e-una-sus>. Acesso em: 30 set. 2015.

BRASIL, Ministério da Saúde. Gabinete do Ministro. Portaria n².436, de 21 de setembro de 2017. Aprova a Política Nacional de Atenção Básica, estabelecendo a revisão de diretrizes para a organização da Atenção Básica, no âmbito do Sistema Único de Saúde (SUS). Diário Oficial da União, Brasília, DF, 22 set. 2017. p. 68.

BRASIL, Ministério da Saúde. 0 SUS de A a Z: garantindo saúde nos municípios. Ministério da Saúde, Conselho Nacional de Secretários Municipais de Saúde, Brasília; 2009. Disponível em: <http://bvsms. saude.gov.br/bvs/publicacoes/Manual_sus_screen.pdf>. Acesso em: 29 abr. 2012.

BRASIL. Ministério da Saúde. Política Nacional de Atenção Básica. Série E. Legislação em Saúde. Secretaria de Atenção à Saúde. Departamento de Atenção Básica. Brasília, 2012; 110 p. Disponível em: <http://189.28.128.100/dab/docs/publicacoes/geral/pnab.pdf>. Acesso em: 15 ago. 2016.

CARNUT, L.; NARVAI, P. C. Avaliação de desempenho de sistemas de saúde e gerencialismo na gestão pública brasileira. Saúde e Sociedade, 25(2), p. 290-305. 2016. Disponível em: <http://www.scielo.br/ scielo.php?script=sci_arttext\&pid=S0104-12902016000200290\&lng=pt\&nrm=iso $>$. Acesso em 01 nov. 2018. 


\section{CONHECIMENTO}

CARRILLO-GARCÍA, C. et al. Influência do gênero e da idade: satisfação no trabalho de profissionais da saúde. Revista Latino-Americana de Enfermagem. 2013; 21(6), 1314-1320. Disponível em: <http://www. redalyc.org/articulo.oa?id=281429401017>. Acesso em: 29 abr. 2015.

CARVALHO, M. S. Programa de Valorização dos Profissionais da Atenção Básica: um olhar implicado sobre sua implantação. 2013. 167 f. Dissertação (Mestrado em Saúde Coletiva) - Universidade de Brasília, Brasília, DF, 2013.

CASTRO, R. C. L. et al. Avaliação da qualidade da atenção primária pelos profissionais de saúde: comparação entre diferentes tipos de serviços. Cad. Saúde Pública. 2012. Disponível em: <http://www. scielo.br/scielo.php?script=sci_arttext\&pid=S0102-311X2012000900015>. Acesso em: 30 jul. 2014.

CHOMATAS, E. et al. Avaliação da presença e extensão dos atributos da atenção primária em Curitiba. Revista Brasileira de Medicina de Família e Comunidade, 2013; [S.I.] 8(29), p. 294-303. Disponível em: <https://www.rbmfc.org.br/rbmfc/article/view/828>. Acesso em: 01 nov. 2017.

CONASS Conselho Nacional de Secretários de Saúde (BR). Para Entender a Gestão do SUS. Brasília: Ministério da Saúde, 2007. 247 p. Disponivel em: <http://bvsms.saude.gov.br/bvs/publicacoes/colec_ progestores_livro8.pdf >. Acesso em: 18 mar. 2013.

COSTA, S.M. et al. Perfil do profissional de nível superior nas equipes da Estratégia Saúde da Família em Montes Claros, Minas Gerais, Brasil. Revista Brasileira de Medicina de Família e Comunidade, [S.I.], 8(27), p. 90-96. 2013. Disponivel em: <http://www.rbmfc.org.br/rbmfc/article/view/530>. Acesso em: 12 nov. 2015.

COTTA, R.M.M. et al. Organização do trabalho e perfil dos profissionais do Programa Saúde da Família: um desafio na reestruturação da atenção básica em saúde. Epidemiol Serv Saúde. v. 15, n. 3, p. 7-18. Disponível em: <http://scielo.iec.pa.gov.br/scielo.php?script=sci_arttext\&pid=S1679-49742006000300002\&lng=pt>. Acesso em: 27 out. 2013.

CRISP, N.; CHEN L. Global supply of health professionals. N Engl J Med. 2013; 370(23), p. 950-7. DOI: 10.1056 / NEJMra1111610. Disponível em: <https://www.nejm.org/doi/full/10.1056/NEJMra1111610>. Acesso em: 09 set. 2018. 


\section{CONHECIMENTO}

DAINESI, S.M.; GOLDBAUM, M. E-survey with researchers, members of ethics committees and sponsors of clinical research in Brazil: an emerging methodology for scientific research. Rev. bras. epidemiol. 2012;15(4), 705-713. Disponível em: <http://www.scielosp.org/pdf/rbepid/v15n4/03.pdf>. Acesso em: 07 jun. 2015.

D'AVILA, L. S. et al. Adesão ao Programa de Educação Permanente para médicos de família de um Estado da Região Sudeste do Brasil. Ciênc. saúde coletiva. 2014; 19(2), p. 401-416. Disponível em: <http://www.scielo.br/scielo.php?script=sci_arttext\&pid=S1413-81232014000200401\&lng=en\&nrm =iso>. Acesso em: 15 Nov. 2018.

DONABEDIAN, A. Evaluating the quality of medical care. Milbank Memorial Fund Q. 2005. Disponivel em: <https://doi.org/10.1111/j.1468-0009.2005.00397.x>. Acesso em: 22 nov. 2015.

FACCHINI, L.A. et al. Performance of the PSF in the Brazilian South and Northeast: institutional and epidemiological Assessment of Primary Health Care. Ciênc. Saúde Coletiva. 2006. Disponivel em: <http://www.scielo.br/pdf/csc/v11n3/30982.pdf>. Acesso em: 29 abr. 2012.

FELISBERTO, E. Monitoramento e avaliação na Atenção Básica: novos horizontes. Revista Brasileira de Saúde Materno Infantil. 2004; 5(7), p. 24-29. Disponivel em: <http://www.scielo.br/pdf/rbsmi/v4n3/ a12v04n3.pdf>. Acesso em: 11 mar. 2012.

FERTONANI, H. P.; et al. The health care model: concepts and challenges for primary health care in Brazil. Ciênc. saúde coletiva. 2015; , 20 (6), p. 1869-1878. Disponível em: <http://www.scielo.br/scielo. php?script=sci_arttext\&pid=S1413-81232015000601869\&Ing=en\&nrm=iso>. Acesso em 09 nov. 2018.

FIÚZA, T.M. et al. Necessidades educacionais dos profissionais da Estratégia Saúde da Família (ESF): possibilidades de Educação em Saúde no município de Fortaleza (CE). Revista Brasileira de Medicina de Família e Comunidade. jun. 2012. [S.I.], v. 7, n. 24, p. 139-146, Disponivel em: <http://www.rbmfc. org.br/rbmfc/article/view/186>. Acesso em: 20 mai. 2013.

GIOVANELLA, L. et al. Saúde da família: limites e possibilidades para uma abordagem integral de atenção primária à saúde no Brasil. Ciência \& Saúde Coletiva. 2009; 14(3), p. 783-794. Disponível em: <http://www.scielo.br/scielo.php?script=sci_arttext\&pid=S1413$81232009000300014 \&$ Ing=pt>. Acesso em: 27 mar. 2012. 


\section{CONHECIMENTO}

GONTIJO, T. L. et al. Avaliação da atenção primária: o ponto de vista de usuários. Saúde debate, 2017; 41(114), p. 741-752, Disponivel em: <http://www.scielo.br/scielo.php?script=sci_arttext\&pid=S0103$11042017000300741 \& \operatorname{lng}=e n \& n r m=i s o>$. Acesso em: 14 nov. 2018.

HARTZ, Z.M.A.; FELISBERTO, E.; SILVIA, L.M.V. Meta-avaliação da atenção básica em saúde: teoria e prática. Rio de Janeiro: Editora Fiocruz. 2008.

MACINKO, J.; STARFIELD, B.; SHI, L. The Contribution of Primary Care Systems to Health Outcomes within Organization for Economic Cooperation and Development (OECD) Countries, 1970-1998. HSR: Health Services Research. 2003; 38(3), p. 831-865. Disponivel em: <http://www.ncbi.nlm.nih.gov/pmc/ articles/PMC1360919/>. Acesso em: 02 mai. 2014.

MATTOS, L. B.; DAHMER, A.; MAGALHÃES, C. R. Contribuição do curso de especialização em Atenção Primária à Saúde à prática de profissionais da saúde. ABCS Health Sciences, v. 40, n. 3. 2015. Disponível em: <https://nepas.emnuvens.com.br/abcshs/article/view/793/688>. Acesso em 08 out. 2018.

MIRANDA, G.R.; OLIVEIRA, G.G.L.; GONÇALVES, M.C. Educação permanente em saúde: dispositivo para a qualificação da Estratégia Saúde da Família. Belém, 2008. Trabalho de Conclusão de Curso (Especialização em Saúde da Família) - Universidade Federal do Pará.

MOTTA, P.R. Desempenho em equipes de saúde - manual. Rio de Janeiro: Opas, Editora FVG, 2001. $135 \mathrm{p}$

OLIVEIRA, M. P. R.; et al. Formação e Qualificação de Profissionais de Saúde: Fatores Associados à Qualidade da Atenção Primária. Rev. bras. educ. med. 2016; 40(4): 547-559. Disponivel em: <http://www. scielo.br/scielo.php?script=sci_arttext\&pid=S0100-55022016000400547\&Ing=pt\&nrm=iso>. Acesso em: 01 nov. 2018.

PINTO, E.S.G.; MENEZES, R.M.P.; VILLA, T.C.S. Situação de trabalho dos profissionais da Estratégia Saúde da Família em Ceará-Mirim. Rev Esc Enferm USP. 2010; 44(3): 657-64. Disponível em: <http://www. scielo.br/pdf/reeusp/v44n3/15.pdf>. Acesso em: 20 mai. 2013.

PIRES, Denise. Reestruturação produtiva e trabalho em Saúde no Brasil. 2. ed. São Paulo: Anna Blume; 2008. 


\section{CONHECIMENTO}

PISCO, L.A. A avaliação como instrumento de mudança. Ciênc. Saúde Coletiva, 2006. Disponível em: <http://www.scielo.br/scielo.php?script=sci_arttext\&pid=S1413-81232006000300004\&nrm=iso>. Acesso em: 20 mar. 2015.

PLATONOW, V. Brasileiros estão casando-se mais tarde, mostra levantamento do IBGE. Agência Brasil, 20/12/2013. Disponivel em: <http://memoria.ebc.com.br/agenciabrasil/noticia/2013-12-20/ brasileiros-estao-casando-se-mais-tarde-mostra-levantamento-do-ibge>. Acesso em: 08 ago. 2015.

PORTELA, G. Z. Atenção Primária à Saúde: um ensaio sobre conceitos aplicados aos estudos nacionais. Physis, v. 27, n. 2, p. 255-276. 2017. Disponível em: <http://www.scielo.br/scielo.php?script=sci_ arttext\&pid=S0103-73312017000200255\&Ing=en\&nrm=iso>. Acesso em: 14 Nov. 2018.

STARFIELD, B.; SHI, L. Policy Relevant Determinants of Health: An International Perspective. Health Policy, v. 60, p. 201-218. 2002. Disponível em: <https://doi.org/10.1016/S0168-8510(01)00208-1>. Acesso em: 02 mai. 2014.

TOMASI, E. et al. Perfil sócio-demográfico e epidemiológico dos trabalhadores da atenção básica à saúde nas regiões Sul e Nordeste do Brasil. Cadernos de Saúde Pública. 24(Suppl. 1), s193-s201. 2008. Disponível em: <http://www.scielo.br/pdf/csp/v24s1/23.pdf>. Acesso em: 9 mar. 2012.

TONELLI, B.; et al. Rotatividade de profissionais da Estratégia Saúde da Família no município de Montes Claros, Minas Gerais, Brasil. Revista Da Faculdade De Odontologia - UPF, v. 23, n. 2, p. 180-185. 2018. Disponível em: <https://doi.org/10.5335/rfo.v23i2.8314>. Acesso em: 30 ago. 2018.

WEYKAMP, J. M. et al. Educação permanente em saúde na atenção básica: percepção dos profissionais de enfermagem. Revista de Enfermagem da UFSM, v. 6, n. 2, p. 281-289. 2018. Disponível em: <https://periodicos.ufsm.br/reufsm/article/view/16754>. Acesso em: 14 nov. 2018.

VIEIRA, H. C.; CASTRO, A. E.; JÚNIOR SCHUCH, V. F. O uso de questionários via e-mail em pesquisas acadêmicas sob a ótica dos respondentes. XIII SEMEAD Seminários em administração. 2010. Disponivel em: <http://www.ead.fea.usp.br/semead/13semead/resultado/trabalhosPDF/612.pdf>. Acesso em: 02 abr. 2016. 
Figura 1: Fluxograma do estudo da coleta e análise dos resultados.

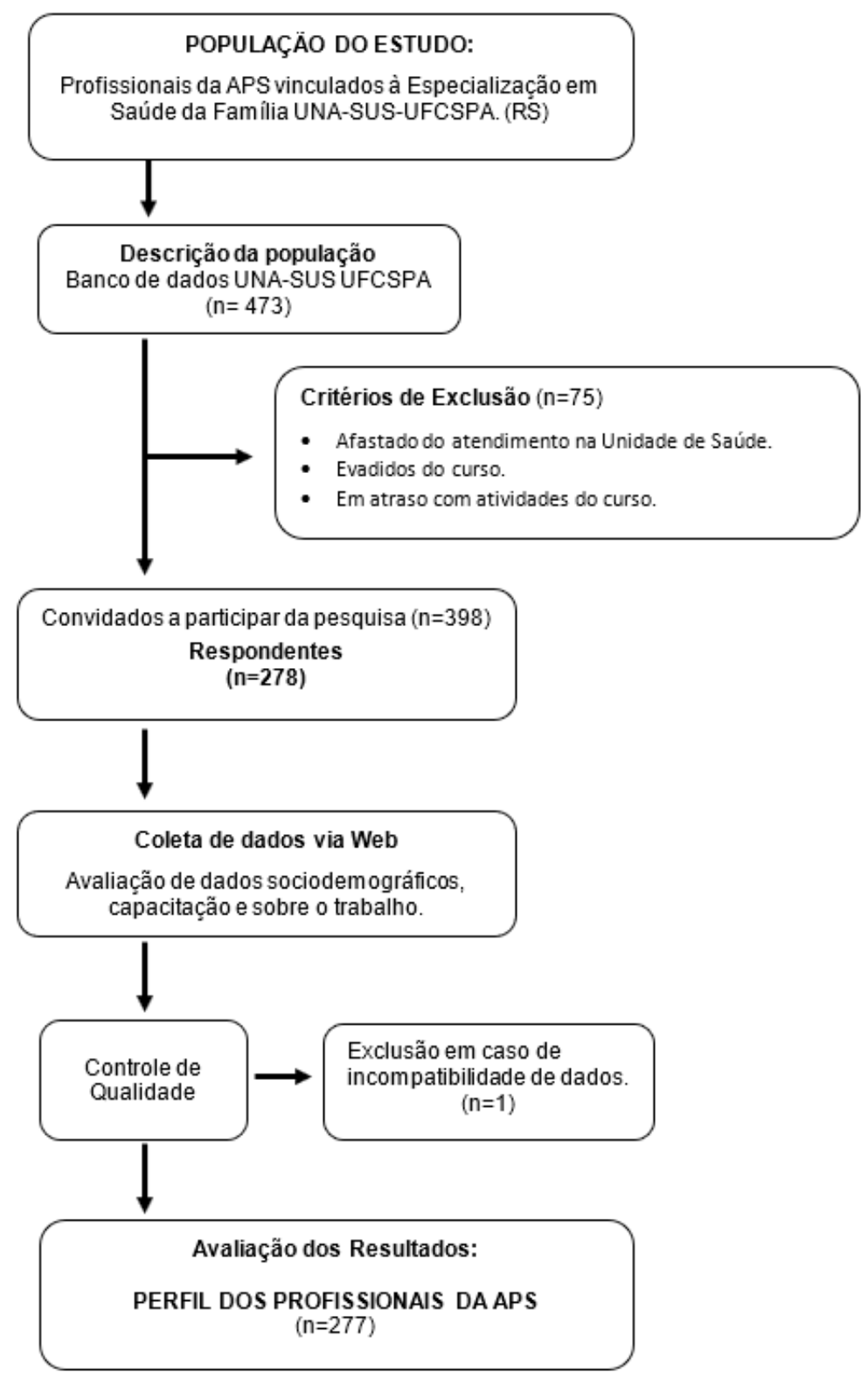

